\title{
Albumin deposition in dermal capillary basement membrane in parents of Type 1 (insulin-dependent) diabetic patients
}

\author{
B. Chavers ${ }^{1}$, D. Etzwiler ${ }^{1}$, J. Barbosa ${ }^{2}$, F. H. Bach ${ }^{3,4}$ and A. F. Michael ${ }^{1,3}$ \\ Departments of ${ }^{1}$ Pediatrics, ${ }^{2}$ Internal Medicine, ${ }^{3}$ Laboratory Medicine and Pathology and \\ ${ }^{4}$ Surgery, University of Minnesota Medical School, Minneapolis, Minnesota, USA
}

\begin{abstract}
Summary. Skin biopsies were performed to examine dermal capillary basement membranes for albumin by immunofluorescence microscopy in children and young adults with Type 1 (insulin-dependent) diabetes from simplex and multiplex families, their non-diabetic parents and control subjects. Circumferential vessel staining for albumin was $12.0 \pm 14.4 \%$ (mean $\pm \mathrm{SD}$ ) in diabetic patients from multiplex families, $18.8 \pm 20.0 \%$ in diabetic patients from simplex families, and $18.5 \pm 24.2 \%$ in unaffected parents from simplex families. These values were significantly higher $(p<0.005)$ than those obtained in unaffected parents from multiplex families $(0.4 \pm 0.9 \%)$ or control subjects $(0.8 \pm 1.7 \%)$. There was no statistically significant difference between Type 1 diabetic pro-
\end{abstract}

bands from simplex or multiplex families and unaffected parents from simplex families. In the simplex families, positive capillary staining for albumin was present only in parents from families in which the diabetic proband had positive staining. In the parents, the presence of dermal capillary staining for albumin did not correlate with an abnormal glucose tolerance test or the presence of cytoplasmic islet cell antibodies. These observations suggest an abnormality in the dermal capillary basement membrane in some unaffected parents of Type 1 diabetic patients from simplex families.

Key words: Albumin, Type 1 diabetes, dermal capillary basement membrane.
Type 1 (insulin-dependent) diabetes appears to be a genetically heterogeneous disease [1-4]. Attempts to identify individuals at increased risk for either developing or transmitting diabetes have been hindered by the lack of definitive biochemical, immunological or histological indicators. Several researchers have suggested that individuals at risk include family members with HLA-DR3 and/or DR4 [2-4], family members with antibodies to surface or cytoplasmic islet cell antigens [5, $6]$, and siblings of a Type 1 diabetic subject $[7,8]$. Previous studies in our laboratory, using immunofluorescence microscopic methods, have demonstrated increased amounts of serum proteins, particularly albumin, in the basement membranes of several organs in Type 1 diabetic patients: kidney $[9,10]$, skeletal muscle sarcolemma and capillaries [11], thyroid gland [12] and dermal capillaries [13]. On the basis of these studies, we attempted to identify tissue indicators of a Type 1 diabetic genetic background. We first tested this possibility by studying non-diabetic siblings of Type 1 diabetic patients. Using immunohistochemical techniques, we found increased staining for albumin in the skeletal muscle sarcolemmal and capillary basement mem- branes in unaffected HLA-identical siblings of diabetic probands [14].

The aim of the present study was to evaluate dermal capillary basement membrane, using immunohistochemical techniques, for the presence of albumin in tissues obtained from unaffected parents from simplex (one child affected but at least two children) and multiplex (two or more siblings affected) Type 1 diabetic families.

\section{Subjects and methods}

\section{Subjects}

We studied 13 diabetic patients from simplex families, aged 8-19 years (mean 13.8 years), 25 unaffected parents from simplex families, aged 32-51 years (mean 41.4 years), 16 diabetic patients from 10 multiplex families, aged $10-34$ years (mean 22.8 years), and 17 unaffected parents from multiplex families, aged 43-66 years (mean 53.1 years) (Table 1). In two of the multiplex families, one parent also had Type 1 diabetes. Twelve of the 13 simplex diabetic patients have been participating in endocrine and immunological studies at the University of Minnesota and were included in a previous report [13]. They did not have evidence of secondary complications of Type 1 dia- 
Table 1. Clinical and laboratory data in subjects studied

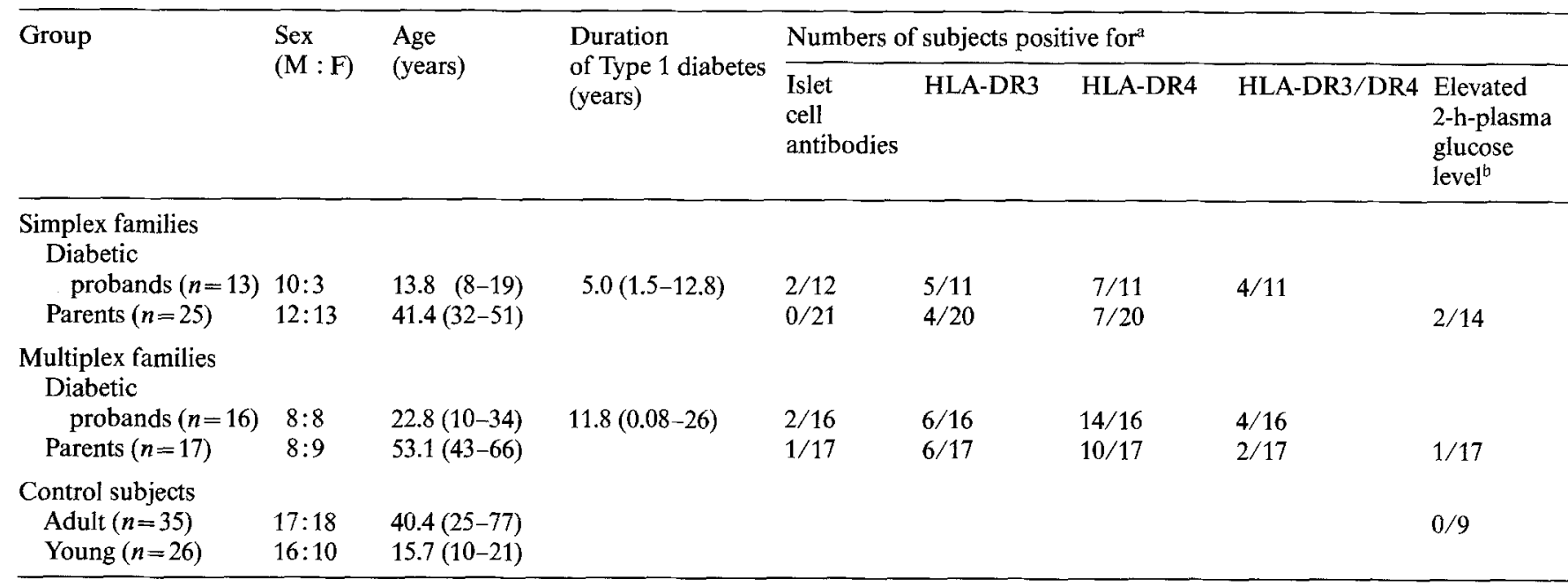

Results expressed as mean with range in parentheses.

a The numerator refers to the number that were abnormal and the denominator the number of individual studies.

There was no significant difference between the simplex and multiplex diabetic patients for mean age of onset of diabetes. However, a significant difference was present for mean duration of diabetes $(p<0.005)$. The mean age of the parental groups differed significantly $(p<0.005)$.

b An elevated 2-h-plasma glucose level $=>7.8 \mathrm{mmol} / 1$

betes (nephropathy or retinopathy). All families were Caucasoid. Members of the control group had negative family histories for diabetes mellitus and were without clinical symptoms of the disease.

The mean age of the 35 adult control subjects was 40.4 years (range 25-77 years) and of the 26 young control subjects was 15.7 years (range 10-21 years). Of the control group, 19 were healthy, normal adult volunteers; the remainder were obtained from patients with non-diabetic diseases, including glomerulonephritis, IgA nephropathy, vasculitis, systemic lupus erythematosus, mixed connective tissue disease, dermatomyositis, psoriasis, familial nephritis, cryoglobulinaemia and systemic Weber-Christian disease. Informed consent was obtained from each subject or from the subject's parents before participation in the study. The studies were approved by the Committee on Use of Human Subjects in Research of the University of Minnesota.

Parents and diabetic patients from multiplex families (except two) were biopsied while fasting and before insulin therapy. Parents and diabetic patients from simplex families were biopsied in the morning under normal conditions (non-fasting, normal activity, 2-4 h after morning insulin therapy) as were the control subjects (except six who were biopsied fasting).

\section{Methods}

Skin biopsies were obtained from the ventral aspect of the right upper arm or the lateral aspect of the right thigh, processed and studied by immunofluorescence using methods described previously [13]. Briefly, stained biopsy tissues from all groups were mixed and read, without knowing their origin, independently by two investigators using an epifluorescence microscope (Zeiss, Oberkochen, FRG) with appropriate fluorescein and rhodamine interference and absorption filters. Each tissue specimen was evaluated for the total number of vessels present and the number of vessels with albumin deposition along the entire circumference of the capillary wall. The percentages of circumferential albumin deposition were determined by counting each vessel present in the biopsy specimen and dividing the total number of vessels with positive circumferential staining for albumin by the total number of vessels present in the section determined by reactivity with rhodamine labelled anti-basement membrane antibody. Vessels with albumin deposition in only a portion of the vessel wall were not considered in determining the percentage of positive vessels. The average number of vessels counted per specimen was 58 (range 16-163).
Oral glucose tolerance tests were performed in 31 parents (14 from simplex families, 17 from multiplex families) and nine healthy adult subjects after 3 days on a $300 \mathrm{~g}$ carbohydrate diet according to the methods described by Fajans and Conn [15]. Plasma glucose levels were measured during these tests. Histocompatibility antigens were determined by standard methods $[16,17]$ in 27 diabetic patients $(11$ from simplex families) and 37 parents ( 20 from simplex families). Cytoplasmic pancreatic islet cell antibodies were determined by indirect immunofluorescence according to published methods [18] in 28 diabetic patients ( 12 from simplex families) and 38 parents ( 21 from simplex families).

Statistical evaluation was carried out by linear regression analyses and Student's t-test. Values for $p$ of 0.05 or less were considered significant.

\section{Results}

\section{Dermal capillary basement membrane immunofluorescence}

Positive staining of the dermal capillary basement membrane (DCBM) for albumin was equally distributed between the superficial and deep dermal vessels. The mean percentage of circumferential DCBM staining for each group was: simplex diabetic patients $18.8 \pm 20.0 \%$ (mean $\pm \mathrm{SD}$ ), multiplex diabetic patients $12.0 \pm 14.4 \%$, parents from simplex families $18.5 \pm 24.2 \%$, parents from multiplex families $0.4 \pm 0.9 \%$, young control subjects $0.6 \pm 2.0 \%$ and adult control subjects $0.9 \pm 1.5 \%$. One adult control with $42 \%$ circumferential staining was excluded from the statistical analysis since this value skewed the results abnormally. There was a significant difference between diabetic (simplex and multiplex) and young control subjects $(p<0.005)$, parents from simplex and multiplex families $(p<0.005)$ and parents from simplex families and adult controls $(p<$ 0.005 ; Fig. 1). There was no statistically significant dif- 


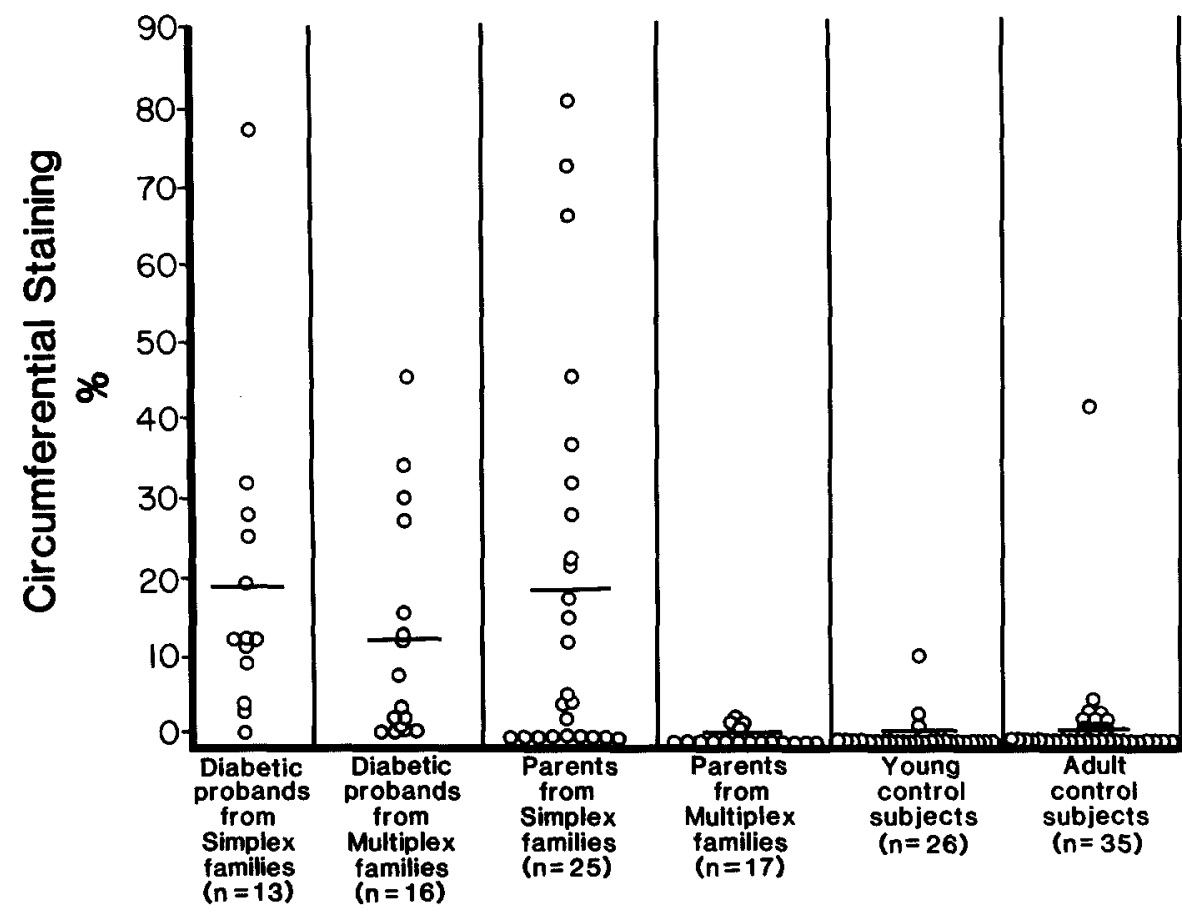

Fig. 1. Percentage circumferential immunofluorescent staining of dermal capillaries for albumin in diabetic probands, parents of diabetic patients and control subjects. Bar indicates mean. Multiplex and simplex diabetic patients were not significantly different, but both were significantly different from young control subjects $(p<0.005)$. Multiplex and simplex parents were significantly different $(p<0.005)$. Multiplex parents did not differ from adult control subjects. However, simplex parents differed from adult control subjects $(p<0.005)$ but not from simplex diabetic probands. There was no significant difference between adult and young control subjects
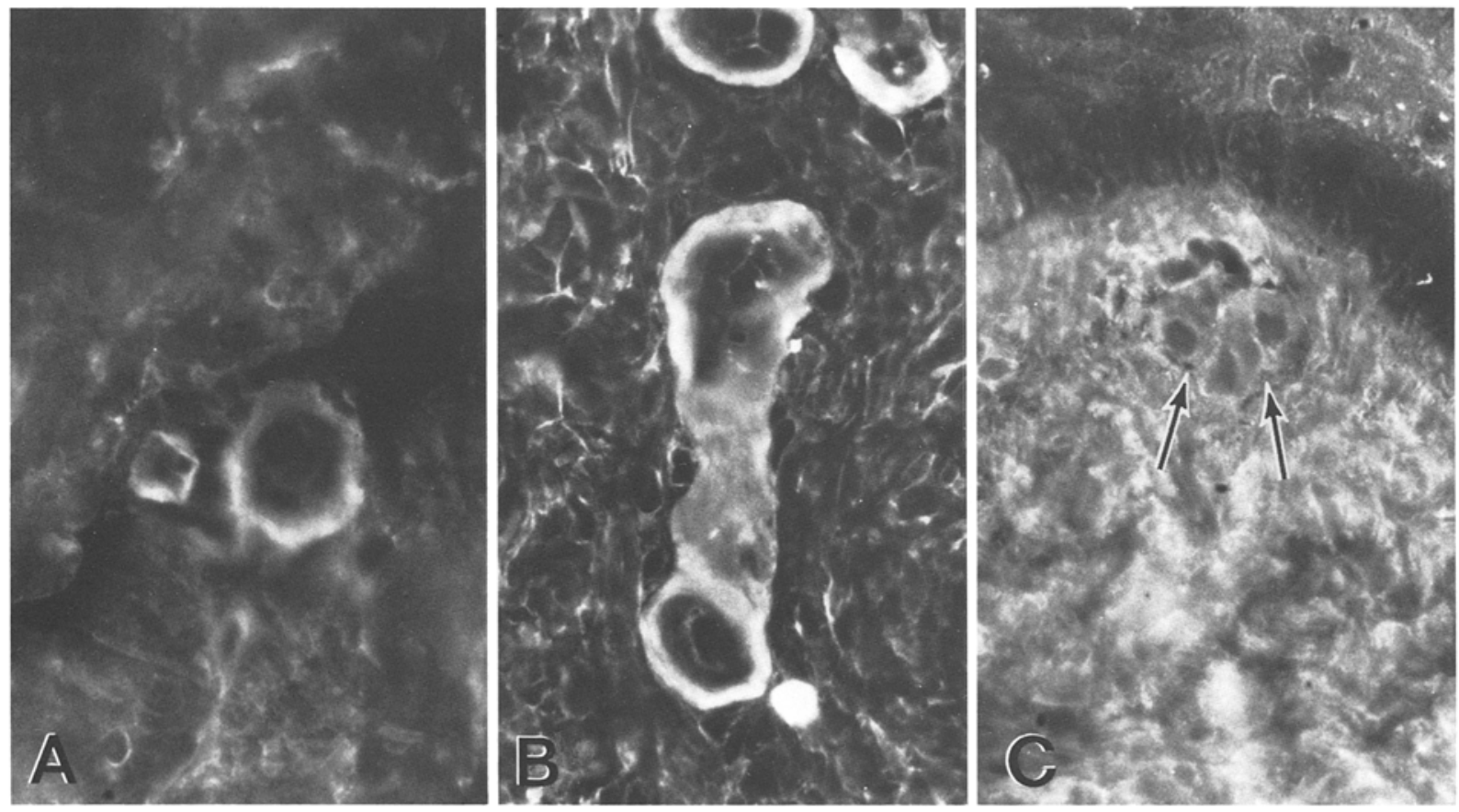

Fig. 2A-C. Skin sections from a simplex family stained with fluorescein isothiocyanate (FITC) conjugated anti-human albumin. Note the fluorescence of the dermal capillary basement membrane (DCBM) for albumin in A the diabetic proband and $\mathbf{B}$ one parent. Note the lack of fluorescence of the DCBM for albumin in the other parent (C, arrows indicate dermal capillaries). All sections were stained sequentially with FITC conjugated anti-human albumin and tetramethyl rhodamine conjugated anti-human basement membrane for unquestionable recognition of the basement membrane of dermal vessels. (X 750)

ference in circumferential DCBM staining between the following groups: simplex and multiplex diabetic patients, parents from simplex families and diabetic patients (simplex, multiplex), and adult versus young control subjects. By linear regression analysis there was no correlation between percentage circumferential staining and age in the diabetic patients $(r=0.35$ multiplex; $r=0.24$ simplex). However, there was a significant correlation between percentage circumferential staining and duration of diabetes for the multiplex diabetic patients $(r=0.63, p=0.009)$ but not for the simplex diabetic patients $(r=0.22)$.

In an attempt to determine whether this finding was an artefact, we repeated biopsies in five Type 1 diabetic 
patients at 1 (four) and 2 (one) years after the initial biopsy. Four were biopsied each time under the same conditions (in the morning, 2-4 $\mathrm{h}$ after insulin therapy) and in one patient the second biopsy was carried out while the patient was fasting and before insulin therapy. The results remained unchanged in each patient (two negative, three positive) even though the sites were changed in two (arm-thigh, arm-abdomen).

In the simplex families, at least one parent of each of the 10 diabetic patients with increased circumferential DCBM staining had increased staining (Fig. 2). In two of these families, both parents were positive. In six of the positive skin biopsies, focal staining along the dermal epidermal junction was present for albumin; this finding was not present in either multiplex parents or control subjects.

\section{Glucose tolerance tests, HLA and pancreatic islet cell antibodies}

Elevated 2-h plasma glucose levels were present in two simplex parents $(8.3$ and $9.7 \mathrm{mmol} / 1)$ and one multiplex parent $(8.9 \mathrm{mmol} / \mathrm{l})$, although no value between 0 and $2 \mathrm{~h}$ was $>11 \mathrm{mmol} / 1$. The glucose tolerance tests were normal in the nine adult control subjects studied.

In the simplex families, HLA-DR3 was present in five diabetic patients and four parents, HLA-DR4 was present in seven diabetic patients and seven parents, and both DR3 and DR4 were present in four diabetic patients. In the multiplex families, HLA-DR3 was present in six diabetic patients and seven parents, HLADR4 was present in 14 diabetic patients and 12 parents, and both DR3 and DR4 were present in four diabetic patients and two parents.

Cytoplasmic pancreatic islet cell antibodies were present in four diabetic patients (two simplex, 2 and 3.8 years after onset of diabetes; two multiplex, 1 month and 3 years after onset of diabetes) and one multiplex parent.

\section{Discussion}

Østerby et al. have suggested that insulin has an acute effect on endothelial cells and increases the transfer of fluid and albumin out of the vascular system [19]; these studies performed in rats demonstrated no differences between insulin-injected diabetic rats and non-diabetic saline-injected control rats [19]. Based on these results, one might expect positive DCBM staining for albumin to be present in diabetic patients biopsied following insulin therapy and in non-fasting control subjects. The results of this study, however, show that circumferential DCBM staining for albumin is a common finding in Type 1 diabetic patients and does not appear to be affected by fasting or the absence of insulin therapy. We also found a significant difference in staining results be- tween diabetic and control subjects irrespective of insulin administration before biopsy.

The presence of albumin in the DCBM did not correlate with either glucose intolerance or islet cell antibodies. Therefore, this phenomenon occurs independently of islet cell failure and cannot be proven by the present studies to be a marker of susceptibility to Type 1 diabetes. It is not unreasonable, however, to speculate that common genetic factors related to Type 1 diabetes are delineated by this histological marker in the parents of Type 1 diabetic patients. However, DCBM albumin localization in parents of Type 1 diabetic subjects is not a generalized finding as evidenced by the lack of positive staining in multiplex parents. Although genetic HLA markers show no distinction between simplex and multiplex families $[4,8,20]$, these studies raise the question of possible differences between the two groups.

Siperstein et al. [21, 22] suggested that basement membrane thickening occurs before glucose intolerance and is a marker of Type 1 diabetes in unaffected first degree relatives of Type 1 diabetic patients. We did not measure DCBM thickness. However, in our previous study, skeletal muscle sarcolemmal and capillary basement membrane staining for albumin was not associated with basement membrane thickening [14]. Preliminary studies performed in our laboratory suggest that this phenomenon occurs as a result of an alteration in one or more structural components of the basement membrane [23]. Here antibody probes were directed against proteins with varying isoelectric points and were used to evaluate glomerular capillary basement membranes by immunofluorescence in kidney tissues obtained from Type 1 diabetic patients and normal human kidneys. Linear staining of the glomerular basement membrane was present in the diabetic tissues only for proteins with an isoelectric point of 6.0 or lower [23] (albumin has an isoelectric point of 4.9). Albumin deposition in DCBM is most likely related to the presence of increased numbers of positive charge sites in the basement membrane which bind this anionic plasma protein in vivo.

Acknowledgements. The technical assistance of K.Divine, L. Lang, K. Pinkham, C. Blocher, E. Van Der Hagen, M. Hoff, D. Cielinski, and the secretarial assistance of C. Dawis are appreciated. This work was supported by NIH grants (AM26149, R01 AI-GM 17687, AM20719, AM01014), NIH contract N01-HD-8-2846, American Diabetes Association Research Grant, the Vikings Children's Fund, the March of Dimes (6-255), and the KROC Foundation, California.

\section{References}

1. National Diabetes Data Group (1979) Classification and diagnosis of diabetes mellitus and other categories of glucose intolerance. Diabetes 28: 1039-1057

2. Cudworth AG (1978) Type I diabetes mellitus. Diabetologia 14: 281-291

3. Rotter JI, Rimoin DL (1981) The genetics of the glucose intolerance disorders. Am J Med 70: 116-126 
4. Barbosa J, Bach FJ, Rich SS (1982) Genetic heterogeneity of diabetes and HLA. Clin Genet 21:25-32

5. Bottazzo GF, Mann JI, Thorogood M, Baum JD, Doniach D (1978) Autoimmunity in juvenile diabetics and their families. $\mathrm{Br}$ Med J 2: 165-168

6. Dobersen MJ, Scharff JE, Ginsberg-Fellner F, Notkins AL (1980) Cytotoxic autoantibodies to beta cells in the serum of patients with insulin-dependent diabetes mellitus. N Engl J Med 303: 1492-1498

7. Barnett AH, Eff C, Leslie RDG, Pyke DA (1981) Diabetes in identical twins: a study of 200 pairs. Diabetologia 20: 87-93

8. Walker A, Cudworth AG (1980) Type I (insulin-dependent) diabetic multiplex families: mode of genetic transmission. Diabetes 29: 1036-1039

9. Westberg NG, Michael AF (1972) Immunopathology of diabetic glomerulosclerosis. Diabetes 21: 163-168

10. Miller K, Michael AF (1976) Immunopathology of renal extracellular membranes in diabetes mellitus: specificity of tubular basement membrane immunofluorescence. Diabetes 25: 701-708

11. Cohn RA, Mauer SM, Barbosa J, Michael AF (1978) Immunofluorescence studies of skeletal muscle extracellular membranes in diabetes mellitus. Lab Invest 39: 13-16

12. Raij J, Michael AF (1981) Immunofluorescence studies of thyroid gland in diabetes mellitus. Lancet 1:671

13. Chavers B, Etzwiler D, Michael AF (1981) Albumin deposition in dermal capillary basement membrane in insulin-dependent diabetes mellitus: a preliminary report. Diabetes 30:275-278

14. Barbosa J, Cohn RA, Chavers B, Michael AF, Steffes M, Hoogwerf B, Szalapski E, Mauer SM (1980) Muscle extracellular membrane immunofluorescence and HLA as possible markers of prediabetes. Lancet 2:330-333

15. Fajans S, Conn J (1965) Prediabetes, sub-clinical diabetes, and latent clinical diabetes: interpretation, diagnosis, and treatment. In: Leibel B, Wrenshall G (eds). On the nature and treatment of diabetes. International Congress Series, Excerpta Medica, New York, pp $641-656$
16. Amos DB, Bashir W, Boyle W, MacQueen M, Tiilikainen A (1969) A simple microcytotoxicity test. Transplantation 7: 200-223

17. Danilovs J, Ayoub G, Terisaki P(1980) Report of the Eighth International Histocompatibility Workshop. In: Terasaki P (ed), Histocompatibility testing 1980. UCLA Tissue Typing Laboratory, Los Angeles, pp 287-288

18. Barbosa J, Chavers B, Dunsworth T, Michael A (1982) Islet cell antibodies and histocompatibility antigens (HLA) in insulindependent diabetics and their first-degree relatives. Diabetes 31 : 585-588

19. Østerby R, Gundersen HJG, Christensen NJ (1978) The acute effect of insulin on capillary endothelial cells. Diabetes 27: 745-749

20. Anderson CE, Hodge SE, Rubin R, Rotter JI, Terasaki PI, Irvine WJ, Rimoin DL (1983) A search for heterogeneity in insulin dependent diabetes mellitus: HLA and autoimmune studies in simplex, multiplex and multigenerational families. Metabolism 32: 471-477

21. Siperstein MD, Unger RH, Madison LL (1968) Studies of muscle capillary basement membranes in normal subjects, diabetic, and prediabetic patients. J Clin Invest 47: 1973-1999

22. Siperstein MD, Raskin P, Burns H (1973) Electron microscopic quantification of diabetic microangiopathy. Diabetes 22: 514-527

23. Melvin T, Kim Y, Michael AF (1983) Selective binding of IgG4 and other negatively-charged proteins in normal and diabetic kidneys. Kidney Int 23: $195 \mathrm{~A}$

Received: 16 May 1983

and in revised form: 21 March 1984

Dr. Blanche Chavers

Department of Pediatrics

University of Minnesota

Box 491 Mayo Memorial Building

420 Delaware Street SE

Minneapolis, MN 55455, USA 